\title{
A Hidden Markov Modeling Approach for Identifying Tumor Subclones in Next-Generation Sequencing Studies
}

\author{
HYOYOUNG CHOO-WOSOBA, PAUL S. ALBERT, BIN ZHU* \\ Biostatistics Branch, Division of Cancer Epidemiology and Genetics, \\ National Cancer Institute, Rockville, MD 20850 \\ bin.zhu@nih.gov
}

\begin{abstract}
Allele-specific copy number alteration (ASCNA) analysis is for identifying copy number abnormalities in tumor cells. Unlike normal cells, tumor cells are heterogeneous as a combination of dominant and minor subclones with distinct copy number profiles. Estimating the clonal proportion and identifying mainclone and subclone genotypes across the genome is important for understanding tumor progression. Several ASCNA tools have recently been developed, but they have been limited to the identification of subclone regions, and not the genotype of subclones. In this paper, we propose subHMM, a hidden Markov model-based approach that estimates both subclone region as well as region-specific subclone genotype and clonal proportion. We specify a hidden state variable representing the conglomeration of clonal genotype and subclone status. We propose a two-step algorithm for parameter estimation, where in the first step, a standard hidden Markov model with this conglomerated state variable is fit. Then, in the second step, region-specific estimates of the clonal proportions are obtained by maximizing region-specific pseudo-likelihoods. We apply subHMM to study renal cell carcinoma datasets in The Cancer Genome Atlas. In addition, we conduct simulation studies that show the good performance of the proposed approach. The $\mathrm{R}$ package is available online at https://dceg.cancer.gov/tools/analysis/subhmm. somatic copy number alteration; tumor heterogeneity; E-M algorithm; forwardbackward algorithm.
\end{abstract}




\section{Introduction}

Somatic copy number alterations (SCNA) are genetic changes in the cancer genome where the copy number of tumor cells departs from two copies through copy number deletion or amplification. SCNA analysis is used to identify genes with an abnormal copy number that contributes to carcinogenesis and cancer progression (Zack et al., 2013).

There are a number of analytical challenges in studying SCNA (Egeblad et al., 2010). First, the average copy number across chromosomes, called ploidy, may not be two and has to be estimated and adjusted for in the analysis. Second, there are various types of SCNA, some of which share the same total copy number. To distinguish them, allele-specific copy number alteration (ASCNA) analysis is preferred, which infers both the total copy number and the minor copy number in each locus. ASCNA analysis is essential in identifying copyneutral LOH (loss of heterozygosity), in which one of the heterogeneous alleles is lost and the other is duplicated. (e.g., the genotype altering from $\mathrm{AB}$ to $\mathrm{AA}$ with the total copy number unchanged). Third, tumor tissue generally contains a mixture of tumor and normal cells; this type of heterogeneity, called tumor purity, needs to be accounted for to obtain correct inference about ASCNA. Finally, tumor cells themselves are heterogeneous with distinct copy number profiles reflecting a dominant clone (mainclone) and minor clones (subclone). Identifying main- and sub-clonal ASCNA across the genome would help design targeted therapeutics against genes with SCNA in both mainclone and subclone.

Several ASCNA tools, such as ASCAT (Van Loo et al., 2010), GPHMM (Li et al., 2011), MixHMM (Liu et al., 2010), and hsegHMM (Choo-Wosoba et al., 2018) have been developed that account for both tumor purity and ploidy. However, these methods assume that the tumor is homogeneous and consists of only a single clone. Tumor heterogeneity has been addressed by a number of authors ((Ha et al., 2014),(Shen and Seshan, 2016), (Li and Xie, 2015)), but no methods have been developed to infer multi-clonal genotype. We propose subHMM based on a hidden Markov model, that identifies multiple clones cross the genome. Standard HMM modeling has been used for both germline and so- 
matic copy number variation (CNV) analysis ((Titsias et al., 2016), (Fan et al., 2017), (Cheng et al., 2017), (Yau et al., 2011), etc). In these cases, the forwardbackward algorithm (Baum, 1972) with an E-M algorithm can be applied so that maximum-likelihood estimation is feasible. For the current problem where a clonal-specific genotype need to be identified across the genome, the forwardbackward algorithm can not be used since the E-step involves computing conditional expectations of complex functions of sequential subclone indicators. Instead, a novel two-step algorithm is proposed for parameter estimation.

The remaining of the paper is organized as follows. In Section 2, we propose subHMM based on a hidden Markov model through the proposed two-step estimation procedure for inference. In Section 3, we apply subHMM to whole exome sequencing (WES) renal cell carcinoma data in the Cancer Genome Atlas (TCGA) project. We perform simulation studies to demonstrate the utility of the approach in Section 4. A discussion follows in Section 5.

\section{Hidden Markov Model for Subclone: subHMM}

Let $W_{k}$ be a hidden state of the $k$ th locus representing mainclone genotype, subclone status, and subclone genotype, where $W_{k}$ follows a Markov chain with a transition probability $A: A_{w^{\prime} w}=P\left(W_{k}=w \mid W_{k-1}=w^{\prime}\right)$ for $k=1, \cdots, N$ with the number of loci, $N$. More formally, we decompose $W_{k}$ into three different variables: $Z_{k}, U_{k}$, and $T_{k} . Z_{k}$ indicates the mainclone genotype of the $k$ th locus; $U_{k}$ is the indicator of whether subclone exists at the $k$ th locus; $T_{k}$ is the subclone genotype when subclone exists. Figure 1 provides a schematic diagram detailing the transitions of $W_{k}$ across consecutive loci.

We consider genotypes (described in Web Appendix A) up to total copy number five. The hidden state space indicator $W_{k}=\left\{Z_{k}, U_{k}, T_{k}\right\}$ includes these twelve different mainclone genotypes, $Z_{k} \in G=\{0, A, \cdots, A A A A A\}$, the indicator of a subclone $U_{k} \in\{0,1\}$, and eleven possible subclone genotypes, $T_{k} \in$ $\left\{G ; T_{k} \neq Z_{k}\right\}$ when a subclone exists $\left(U_{k}=1\right)$. For instance, $Z_{k}=A, U_{k}=1$, and $T_{k}=A A B$ indicates that the mainclone genotype is $A$ with the subclone genotype $A A B ; Z_{k}=A A A$ and $U_{k}=0$ means the mainclone genotype $A A A$ 
with no subclone at the $k$ th locus. Note that $T_{k}$ is not defined when $U_{k}=0$.

For the subclone genotype, we assume that the subclone genotype $T_{k}$ is different from $Z_{k}$, the mainclone genotype, and $T_{k}$ follows a multinomial distribution. Then, the transition probability $A$ can be expressed as

$$
\begin{aligned}
A: A_{\left\{z^{\prime}, u^{\prime}, t^{\prime}\right\}\{z, u, t\}} & =P\left(Z_{k}=z, U_{k}=u, T_{k}=t \mid Z_{k-1}=z^{\prime}, U_{k-1}=u^{\prime}, T_{k-1}=t^{\prime}\right) \\
& =P\left(Z_{k}=z \mid Z_{k-1}=z^{\prime}\right) \times P\left(U_{k}=u \mid U_{k-1}=u^{\prime}\right) \times P\left(T_{k}=t \mid U_{k}=1\right)^{I\left(U_{k}=1, T_{k} \neq z\right)} \\
& =P_{z^{\prime} z}^{Z} \times P_{u^{\prime} u}^{U} \times\left(P_{t}^{T}\right)^{I\left(U_{k}=1, T_{k} \neq z\right)},
\end{aligned}
$$

where $P_{z^{\prime} z}^{Z}$ and $P_{u^{\prime} u}^{U}$ are elements in the probability transition matrices for $Z_{k}$ and $U_{k}$, and $P_{t}^{T}$ is the probability of $T_{k}=t$ where $t \neq z$.

In practice, we do not observe the hidden states, rather we observe allelespecific measurements of $\log \mathrm{R}$ and $\log \mathrm{OR}$, where $\log \mathrm{R}$ characterizes the ratio of total copy numbers in tumor vs normal and $\log \mathrm{OR}$ measures the odds ratio of copy numbers from maternal and paternal alleles in tumor vs normal. Consequently, these measurements depend on mainclone genotype as well as the status of subclones and their genotypes, detailed below.

Let $Y_{k}$ and $X_{k}$ be the $k$ th observation of $\log \mathrm{R}$ and $\log \mathrm{OR}$, respectively. Then, given the hidden states, we specify the conditional distributions of $Y_{k}$ and $X_{k}$, separately. For $Y_{k}$, we use a t-distribution with degrees of freedom to account for hypersegmentation that is common in next-generation sequencing (NGS)-based data ((Peel and McLachlan, 2000),(Choo-Wosoba et al., 2018)). Specifically, we use a normal-gamma mixture to derive the t-distribution with degrees of freedom (i.e. we assume $Y_{k} \sim N\left(\mu, \kappa^{2} / \delta\right)$ with $\delta \sim \operatorname{gamma}\left(\frac{v}{2}, \frac{v}{2}\right)$ where $Y_{k}$ has a marginal expectation of $\mu$ with a variance of $\left.\kappa^{2} \times \frac{v}{v-2}\right)$. We assume that the squared $\log \mathrm{OR}, X_{k}^{2}$, follows a non-central chi-square distribution with one degree of freedom and a non-central parameter $\eta=\lambda^{2} / \sigma^{2}$, where $\lambda$ and $\sigma^{2}$ are the mean and variance of $\log \mathrm{OR}$. Finally, the expectations of $Y_{k}$ and $X_{k}^{2}$ are 
defined as

$$
\mu=E\left(Y_{k} \mid Z_{k}=z, U_{k}=u, T_{k}=t\right)= \begin{cases}\log _{2} \frac{2(1-\alpha)+C_{z} \alpha}{\psi}, & u=0 \\ \log _{2} \frac{2(1-\alpha)+C_{z} M_{r} \alpha+C_{t}\left(1-M_{r}\right) \alpha}{\psi}, & u=1\end{cases}
$$

and

$$
\lambda=E\left(X_{k} \mid Z_{k}=z, U_{k}=u, T_{k}=t\right)= \begin{cases}\log \frac{(1-\alpha)+m_{z} \alpha}{(1-\alpha)+p_{z} \alpha}, & u=0 \\ \log \frac{(1-\alpha)+m_{z} M_{r} \alpha+m_{t}\left(1-M_{r}\right) \alpha}{(1-\alpha)+p_{z} M_{r} \alpha+p_{t}\left(1-M_{r}\right) \alpha}, & u=1\end{cases}
$$

In Equations 2.1 and $2.2, \alpha$ and $\psi$ are the tumor purity and the average ploidy, respectively. A subclone region is delinerated along the chromosome where $U_{k}=1$ for consecutive loci. The parameter $M_{r}$ indicates the mainclone (or clonal) proportion of tumor cells in the $r$ th subclone region. $C_{z}$ and $C_{t}$ are copy numbers from maternal and paternal alleles, $m_{z}$ and $m_{t}$ are copy numbers of maternal alleles, and $p_{z}$ and $p_{t}$ are copy numbers of paternal alleles, each corresponding to the mainclone (subscript $z$ ) and subclone (subscript $t$ ), respectively. $\alpha, \psi, \kappa^{2}, v$, and $\sigma^{2}$ are global parameters over the entire genome.

Since $\log \mathrm{R}$ and $\log \mathrm{OR}$ values are assumed to be conditionally independent given the states, the joint emission probability is constructed simply by multiplying the two density functions.

$$
\begin{aligned}
f_{\boldsymbol{\theta}_{\boldsymbol{r}}}\left(Y_{k}, X_{k} \mid Z_{k}=z, U_{k}=u, T_{k}=t\right) & =t_{v}\left(Y_{k} \mid Z_{k}=z, U_{k}=u, T_{k}=t, \boldsymbol{\theta}_{\boldsymbol{r}}\right) \\
& \times \chi_{1}^{2}\left(X_{k}^{2} \mid Z_{k}=z, U_{k}=u, T_{k}=t, \boldsymbol{\theta}_{\boldsymbol{r}}\right),
\end{aligned}
$$

where $\boldsymbol{\theta}_{\boldsymbol{r}}=\left\{\alpha, \psi, M_{r}, \kappa^{2}, \sigma^{2}, v\right\}$.

Based on this model structure, we consider two different assumptions about the clonal proportion. First, we assume a constant clonal proportion where all the identified sublone regions have the exact same clonal proportions of tumor cells. Second, we assume that each subclone region has a potentially different clonal proportion $\left(M_{r}\right)$. The first model is consistent with a single subclone, and the second with multiple subclones in the tumor. 


\section{1 subHMM with E-M algorithm under the constant clonal proportion assumption}

We consider the situation where mainclone/subclone proportions are constant across all the subclone regions $\left(M_{r}=M\right)$. Since $W_{k}$ follows an unobserved Markov chain, we can directly apply the E-M algorithm to estimate all the parameters by maximizing the expectation of a complete log likelihood function

$$
Q=E_{\boldsymbol{W} \mid \boldsymbol{Y}, \boldsymbol{X}^{2}, \theta}\left[\log L\left(\theta ; \boldsymbol{Y}, \boldsymbol{X}^{\mathbf{2}}, \boldsymbol{W}\right)\right],
$$

where $\boldsymbol{\theta}_{\boldsymbol{r}}=\left\{\alpha, \psi, M, \kappa^{2}, \sigma^{2}, v\right\}$, and where we denote all the bold characters as vectors, such as $\boldsymbol{Y}=\left\{Y_{1}, Y_{2}, \cdots, Y_{N}\right\}, \boldsymbol{X}^{\mathbf{2}}=\left\{X_{1}, X_{2}, \cdots, X_{N}\right\}$, and $\boldsymbol{W}=$ $\left\{W_{1}, W_{2}, \cdots, W_{N}\right\}$. Rewriting $\boldsymbol{W}$ with respect to $\boldsymbol{Z}, \boldsymbol{U}$, and $\boldsymbol{T}$, our expectation of the complete $\log$ likelihood function $Q$ is then redefined as,

$$
\begin{aligned}
Q & =\sum_{z \in G} \sum_{u} \sum_{t \in G ; t \neq z} \gamma_{1}(z, u, t) \times\left\{\log P\left(Z_{1}=z\right)+\log P\left(U_{1}=u\right)+I(u=1) \times \log P_{t}^{T}\right\}+ \\
& \sum_{k=2}^{N} \sum_{z \in G} \sum_{u} \sum_{t \in G ; t \neq z} \sum_{z^{\prime} \in G} \sum_{u^{\prime}} \eta_{k-1, k}\left(z^{\prime}, u^{\prime}, t^{\prime} ; z, u, t\right) \times\left(\log P_{z^{\prime} z}^{Z}+\log P_{u^{\prime} u}^{U}+\log P_{t}^{T}\right)+ \\
& \sum_{k=1}^{N} \sum_{z \in G} \sum_{u} \sum_{t \in G ; t \neq z} \gamma_{k}(z, u, t) \times \log f_{\theta}\left(Y_{k}, X_{k}^{2} \mid Z_{k}=z, U_{k}=u, T_{k}=t\right) \\
& =Q_{1}+Q_{\eta}+Q_{\theta},
\end{aligned}
$$

where $\gamma_{k}(z, u, t)=P\left(Z_{k}=z, U_{k}=u, T_{k}=t \mid \boldsymbol{Y}, \boldsymbol{X}^{\mathbf{2}}\right)$ and $\eta_{k-1, k}\left(z^{\prime}, u^{\prime}, t^{\prime} ; z, u, t\right)=$ $P\left(Z_{k}=z, U_{k}=u, T_{k}=t, Z_{k-1}=z^{\prime}, U_{k-1}=u^{\prime}, T_{k-1}=t^{\prime} \mid \boldsymbol{Y}, \boldsymbol{X}^{\mathbf{2}}\right)$ and importantly, the indicators are sufficient statistics for initial probabilities, transition probabilities, and emission probability.

In the E-step, we obtain $\gamma_{k}(z, u, t)$ and $\eta_{k-1, k}\left(z^{\prime}, u^{\prime}, t^{\prime} ; z, u, t\right)$ with the forwardbackward algorithm introduced by Baum (1972). The forward algorithm starts with calculating the forward element of the first loci measurements, $a_{w}(1)$, de- 
composed to $Z, U$, and $T$, following by

$$
\begin{aligned}
a_{w}(1)=a_{z, u, t}(1) & =P\left(Y_{1}, X_{1}^{2}, Z_{1}=z, U_{1}=u, T_{1}=t\right) \\
& =f_{\theta}\left(Y_{1}, X_{1}^{2} \mid Z_{1}=z, U_{1}=u, T_{1}=t\right) P\left(Z_{1}=z\right) P\left(U_{1}=u\right)\left(P_{t}^{T}\right)^{I\left(u_{k}=1, T_{k} \neq z\right)} .
\end{aligned}
$$

Based on $a_{z, u, t}(1)$, the forward algorithm obtains sequential elements corresponding to each of $k$ locus given by

$$
a_{z, u, t}(k)=\sum_{z^{\prime}, u^{\prime}, t^{\prime}} a_{z^{\prime}, u^{\prime}, t^{\prime}}(k-1) P_{z^{\prime} z}^{Z} P_{u^{\prime} u}^{U}\left(P_{t}^{T}\right)^{I\left(U_{k}=1, T_{k} \neq z\right)} f_{\theta}\left(Y_{k}, X_{k}^{2} \mid Z_{k}=z, U_{k}=u, T_{k}=t\right)
$$

The backward element of the $k$ th locus is defined as

$$
\begin{aligned}
b_{z, u, t}(k) & =P\left(Y_{k+1}, Y_{k+2}, \cdots, Y_{N}, X_{k+1}^{2}, X_{k+2}^{2}, \cdots, X_{N}^{2} \mid Z_{k}=z, U_{k}=u, T_{k}=t\right) \\
& =\sum_{z^{\prime}, u^{\prime}, t^{\prime}} b_{z^{\prime}, u^{\prime}, t^{\prime}}(k+1) P_{z^{\prime} z}^{Z} P_{u u^{\prime}}^{U}\left(P_{t^{\prime}}^{T}\right)^{I\left(u_{k+1}=1, T_{k+1} \neq z^{\prime}\right)} f_{\theta}\left(Y_{k+1}, X_{k+1}^{2} \mid Z_{k+1}=z^{\prime}, U_{k+1}=u^{\prime}, T_{k+1}=t^{\prime}\right) .
\end{aligned}
$$

The backward algorithm starts with calculating the backward element of the last locus, $b_{z, u, t}(N)$ defined as 1 for all the hidden states, and obtains all the backward elements recursively. Finally, we can compute $\gamma_{k}(z, u, t)$ and $\eta_{k-1, k}\left(z^{\prime}, u^{\prime}, t^{\prime} ; z, u, t\right)$ by using both forward and backward elements, given by

$$
\gamma_{k}(z, u, t)=\frac{a_{z, u, t}(k) b_{z, u, t}(k)}{\sum_{z^{\prime}, u^{\prime}, t^{\prime}} a_{z^{\prime}, u^{\prime}, t^{\prime}}(N)}
$$

and

$\eta_{k-1, k}\left(z^{\prime}, u^{\prime}, t^{\prime} ; z, u, t\right)=\frac{P_{z^{\prime} z}^{Z} P_{u^{\prime} u}^{U}\left(P_{t}^{T}\right)^{I(u=1, t \neq z)} a_{z^{\prime}, u^{\prime}, t^{\prime}}(k-1) b_{z, u, t}(k) f_{\theta}\left(Y_{k}, X_{k}^{2} \mid Z_{k}=z, U_{k}=u, T_{k}=t\right)}{\sum_{z^{\prime}, u^{\prime}, t^{\prime}} a_{z^{\prime}, u^{\prime}, t^{\prime}}(N)}$,

which are both sufficient statistics of $Q$ (Equation 2.4) under the constant $M$ assumption.

In E-step, given $\gamma_{k}(z, u, t)$ and $\eta_{k-1, k}\left(z^{\prime}, u^{\prime}, t^{\prime} ; z, u, t\right)$, we can finally estimate all the parameters by maximizing $Q$. First, we estimate $P\left(Z_{1}=z\right), P_{z^{\prime} z}^{Z}, P_{u^{\prime} u}^{U}$, 
and $P_{t}^{T}$ by closed forms. For $P\left(Z_{1}=z\right)$,

$$
\frac{\partial Q}{\partial P\left(Z_{1}=z\right)}=\frac{\partial Q_{1}}{\partial P\left(Z_{1}=z\right)}=\sum_{u=0}^{1} \sum_{t ; t \neq z} \frac{\gamma_{1}(z, u, t)}{P\left(Z_{1}=z\right)}=0
$$

with respect to $\sum_{z=0}^{11} P\left(Z_{1}=z\right)=1$. According to Lagrangian multipliers,

$$
\hat{P}\left(Z_{1}=z\right)=\frac{\sum_{u=0}^{1} \sum_{t ; t \neq z} \gamma_{1}(z, u, t)}{\sum_{z^{*} \in G} \sum_{u=0}^{1} \sum_{t ; t \neq z^{*}} \gamma_{1}\left(z^{*}, u, t\right)}
$$

Similarly, for $P\left(U_{1}=u\right)$,

$$
\hat{P}\left(U_{1}=u\right)=\frac{\sum_{z \in G} \sum_{t ; t \neq z} \gamma_{1}(z, u, t)}{\sum_{u^{*}=0}^{1} \sum_{z \in G} \sum_{t ; t \neq z} \gamma_{1}\left(z, u^{*}, t\right)} .
$$

For estimating $P_{z^{\prime} z}^{Z}, P_{u^{\prime} u}^{U}$, and $P_{t}^{T}$,

$$
\begin{gathered}
\hat{P}_{z^{\prime} z}^{Z}=\frac{\sum_{k=2}^{N} \sum_{u=0}^{1} \sum_{t ; t \neq z} \sum_{u^{\prime}=0}^{1} \sum_{t^{\prime} ; t^{\prime} \neq z^{\prime}} \eta_{k-1, k}\left(z^{\prime}, u^{\prime}, t^{\prime} ; z, u, t\right)}{\sum_{k=2}^{N} \sum_{z^{*} \in G} \sum_{u=0}^{1} \sum_{t ; t \neq z^{*}} \sum_{u^{\prime}=0}^{1} \sum_{t^{\prime} ; t^{\prime} \neq z^{\prime}} \eta_{k-1, k}\left(z^{\prime}, u^{\prime}, t^{\prime} ; z^{*}, u, t\right)}, \\
\hat{P}_{u^{\prime} u}^{U}=\frac{\sum_{k=2}^{N} \sum_{z \in G} \sum_{t ; t \neq z} \sum_{z^{\prime} \in G} \sum_{t^{\prime} ; t^{\prime} \neq z^{\prime}} \eta_{k-1, k}\left(z^{\prime}, u^{\prime}, t^{\prime} ; z, u, t\right)}{\sum_{k=2}^{N} \sum_{z \in G} \sum_{u^{*}=0}^{1} \sum_{t ; t \neq z} \sum_{z^{\prime} \in G} \sum_{t^{\prime} ; t^{\prime} \neq z^{\prime}} \eta_{k-1, k}\left(z^{\prime}, u^{\prime}, t^{\prime} ; z, u^{*}, t\right)}, \text { and }
\end{gathered}
$$

$$
\hat{P}_{t}^{T}=\frac{\sum_{z \in G ; z \neq t} \gamma_{1}(z, u=1, t)+\sum_{k=2}^{N} \sum_{z \in G ; z \neq t} \sum_{z^{\prime} \in G} \sum_{u^{\prime}=0}^{1} \sum_{t^{\prime} ; t^{\prime} \neq z^{\prime}} \eta_{k-1, k}\left(z^{\prime}, u^{\prime}, t^{\prime} ; z, u=1, t\right)}{\sum_{t^{*}} \sum_{z \in G ; z \neq t^{*}} \gamma_{1}\left(z, u=1, t^{*}\right)+\sum_{k=2}^{N} \sum_{t^{*}} \sum_{z \in G ; z \neq t^{*}} \sum_{z^{\prime} \in G} \sum_{u^{\prime}=0}^{1} \sum_{t^{\prime} ; t^{\prime} \neq z^{\prime}} \eta_{k-1, k}\left(z^{\prime}, u^{\prime}, t^{\prime} ; z, u=1, t^{*}\right)} .
$$

We iterate this E-M procedure until convergence to obtain the final global estimates. Specifically, all the global parameters including $M$ are estimated by using optim function in $\mathrm{R}$ for the M-step and the forward-backward algorithm for the E-step. Mainclone genotypes are identified by choosing the maximum posterior probability at each locus. Subclone regions are identified by consecutive loci for which a state with a subclone $\left(U_{k}=1\right)$ obtains the maximum posterior probability. To estimate the region-specific subclone genotype distribution, for each 
subclone region, we average $\gamma_{k}(z, u=1, t)$, the posterior probabilities of each subclone genotype over all the possible mainclone genotypes with the subclone genotype. We determine the subclone genotype at the $r$ th region by choosing the genotype with the largest average posterior probability.

\section{2 subHMM with a two-step algorithm under the differ- ent clonal proportion assumption}

subHMM that incorporates a varying clonal proportion could be formulated with an emission distribution, $f_{\theta}$ (the third line in Equation 2.4), that depends on a region-specific clonal proportion. However, the complete data likelihood would be a complex function of the sequence of subclonal latent variables, $U_{k}$. Because of this, a forward-backward algorithm cannot be implemented, making E-step computations in the E-M algorithm intractable. Hence, we propose a two-step algorithm that accounts for different clonal proportion across subclonal regions.

\subsubsection{The first step}

We first assume that the clonal proportion is constant across subclone regions, $M_{r}=M$. Under this constant clonal proportion assumption, we estimate the global parameters, the probability transition matrix corresponding to the mainclone, and identify the subclone regions as described in Section 2.1.

\subsubsection{The second step}

For each subclone region (identified in the first step), we estimate region-specific clonal proportions and subclone genotype probability distribution. The clonal proportion of the $r$ th subclone region, $\widehat{M}_{r}$ is estimated by locally maximizing the conditional expectation of the log likelihood function for the $r$ th region $R_{r}$, given all the global parameters estimated at the first step:

$\widehat{M}_{r}=\max _{M_{r}}\left\{\sum_{k: k \in R_{r}} \sum_{z \in G} \sum_{u=0}^{1} \sum_{t: t \neq z} \widehat{\gamma}_{k}(z, u, t) \times \log f_{\theta_{r}}\left(Y_{k}, X_{k}^{2} \mid Z_{k}=z, U_{k}=u, T_{k}=t\right)\right\}$. 


\subsection{Asymptotic standard errors of global estimators}

The Hessian-based standard errors require calculating the full log likelihood, which is only obtainable under the constant clonal proportion assumption. For the case of different clonal proportions, we can only compute the log likelihood in the first step of the two-step algorithm under the constant clonal assumption. Therefore, it is important to justify the use of Hessian-based standard errors under the assumption of different clonal proportions in subclone regions (see details in Section 2.2).

Under the constant clonal assumption, the log likelihood function given the estimates is then able to be obtained from the forward-backward algorithm (Section 2.1) by $\log L\left(\boldsymbol{Y}, \boldsymbol{X}^{\mathbf{2}} \mid \hat{\theta}\right)=\log \left(\sum_{z, u, t} a_{z, u, t}(N)\right)$, where $a_{z, u, t}(N)$ is the forward function of the last data point at each hidden state. Then, the asymptotic standard errors of the global estimators, based on the Hessian matrix is calculated as

$$
\mathrm{SE}_{\mathrm{H}}=\sqrt{\operatorname{diag}\left[-\left(\frac{\partial^{2} \log L\left(\boldsymbol{Y}, \boldsymbol{X}^{\mathbf{2}} \mid \hat{\theta}\right)}{\partial \widehat{\boldsymbol{B}} \partial \widehat{\boldsymbol{B}}^{T}}\right)^{-1}\right]},
$$

where $\boldsymbol{B}=\left(\alpha, \psi, \kappa^{2}, v, \sigma^{2}\right)^{T}$ is the vector of global parameters, and the Hessian-based standard error $\mathrm{SE}_{\mathrm{H}}$ can be implemented with Hessian function in the $\mathrm{R}$ package numDeriv.

\section{Analysis of Renal Cell Carcinoma Data}

Initially, we apply subHMM to a single renal cell carcinoma sample with ID, TCGA-KL-8331 from TCGA project (https://cancergenome.nih.gov). TCGA project holds abundant cancer molecular profiling information to understand cancer genomics from a molecular perspective, including large-scale genome sequencing data. The whole exome sequencing dataset of TCGA-KL-8331 is available at https://portal.gdc.cancer.gov, that contains read counts and read depths over entire chromosomes for both normal and tumor samples within the same patient. We use preprocessing codes in FACETS (Shen and Seshan, 
2016) (preProcSample, and procSample) to obtain $\log \mathrm{R}$ and $\log \mathrm{OR}$ measurements from the read-based dataset. A thinning procedure is then conducted by keeping every 10 th values of $\log \mathrm{R}$ and $\log \mathrm{OR}$ to reduce excessive numbers of copy number changes caused by sequencing artefacts (hypersegmentation) and to reduce the computation burden, resulting in 36,914 pairs of $\log \mathrm{R}$ and $\log \mathrm{OR}$. Recalling the definition of $\log \mathrm{OR}$, if a locus is homozygous, the odds ratio is 0 and $\log \mathrm{OR}$ is undefined and only $\log \mathrm{R}$ measurements contribute the estimation of ASCNA. For the sample TCGA-KL-8331, 4,691 (approximately $13 \%$ of 36,914 pairs) loci are heterozygous across the whole chromosomes, for which $\log \mathrm{OR}$ values are defined.

Figure 2 shows the copy number profile of all the chromosomes based on subHMM with the hidden state space reflecting copy numbers of up to 5 . Figure 2.a presents $\log \mathrm{R}$ (the top plot) and $\log \mathrm{OR}$ (the bottom plot) values across the genome with blue dots. The red lines indicate the estimated segment mean of $\log \mathrm{R}$ and $\log \mathrm{OR}$ based on subHMM. Figure 2.b shows estimated clonal profiles of mainclone genotypes (the top plot), subclone regions (the middle plot), and subclone genotypes (the bottom plot). The most frequent identified mainclone genotypes are $\mathrm{A}$ (deletion event) and $\mathrm{AB}$ (normal status) across the genome. Figure 2.b shows 5 subclone regions identified at chromosomes 11, 18, 22, and chromosome $\mathrm{X}$. The subclone regions are also shown as green dots in Figure 2.a. The subclone genotype distributions for regions 1 to 4 suggest that the subclone genotypes are equally likely to be $\mathrm{AB}$ or $\mathrm{AABB}$; No genotype is clearly favored for region 5 (Table B.1 in Appendix B of the Supplementary Materials).

Table 1 summarizes the result of both the global parameter and clonal proportion parameter estimation. Specifically, the estimated purity $\hat{\alpha}$ is 0.91 , which indicates that the tumor sample contains $91 \%$ tumor cells. The estimated ploidy below $2(\hat{\psi} \approx 1.61)$ refers to partial loss of chromosomes in the tumor sample. The clonal proportions obtained by Equation 2.5 for each of the identified subclone regions, $M_{1}$ to $M_{5}$ are also presented in Table 1. Since the region-specific clonal proportions are estimated by maximizing a localized likelihood, Hessianbased asymptotic standard error cannot easily be obtained. Instead, we propose a parametric bootstrap for the estimation of these standard errors. First, we 
simulate a sample under the model using the estimates as known parameters. Then, we fit subHMM to the simulated data. Standard errors for the estimated clonal proportions $\left(\mathrm{SE}_{\mathrm{bs}}\right.$ in Table 1) are obtained by repeating the bootstrap procedure 300 times, and obtaining the empirical standard deviations of the clonal proportion estimates. All the five estimated clonal proportions including the constant proportion $M$ (obtained in the first step) range from $84 \%$ to $89 \%$, which suggests that the proportion of subclone cells in tumor cells ranges between $11 \%$ to $16 \%$.

As shown in Figure 2.b, the predominant (main) genotypes are A and AB, suggesting that the state-space of the HMM may be reduced to (0, A, AA, AB) for both the mainclone and subclone genotypes, corresponding to a maximum copy number of two. Penalized likelihood techniques such as AIC and BIC (shown Table B.3 in Appendix B of the Supplementary Materials) show that a reduced model with a maximum copy number of 2 for both the main-and sub-clone genotypes is favored over models with a maximum copy number of 5. Figure 3 shows four subclone regions identified with this reduced model. These regions are similar to those identified with the complex model with the exception of an additional region identified on chromosome 5 and the lack of a region on chromosome $\mathrm{X}$. The region-specific subclone genotypes presented in the Appendix B of the Supplementary Materials (Table B.2) suggests that the regions $R_{2}$ to $R_{4}$ have an $\mathrm{AB}$ genotype with high confidence and the region $R_{1}$ has a genotype of $\mathrm{A}$ or AA with equal confidence.

Estimated global parameters for the reduced model are presented in Table 2. These estimates are similar to those estimated for the more complex model (Table 1). In contrast with the global parameters, the region-specific clonal proportions (presented in Table 2) show more variation between regions than those estimated with the more complex model. The clonal proportion estimates $M_{3}$ and $M_{4}$, have larger standard errors than those estimated for $M_{1}$ and $M_{2}$, possibly due to their regions being shorter in length. The estimates of $M_{1}$, $M_{2}, M_{3}$, and $M_{4}$ along with their associated standard errors (obtained with the bootstrap) suggest that there may be two underlying clonal proportions; one at 0.7 and the other 0.8 . These two distinct values suggest that there are two 
subclone events in this TCGA-KL-8331 sample. Further evidence of at least two subclones is obtained with the use of a bootstrap Wald test (null hypothesis of constant clonal proportion) that was highly significant $(p<0.0001)$. This new insight on region-specific clonal proportions and genotype is not available with other approaches ((Shen and Seshan, 2016): Appendix C of the Supplementary Materials shows that the subclone regions and mainclone genotypes are similar to those obtained with our approach).

In practice, it is of interest to apply subHMM to a number of samples in order to characterize recurrent mainclone or subclone SCNA events across samples. We apply subHMM to 316 renal cell carcinoma samples from TCGA. Using AIC, we compared subHMM with a model hsegHMM that assumes the existence of mainclone only in tumor sample (Choo-Wosoba et al., 2018); 120 of 316 showed a lower AIC for subHMM compared to hsegHMM, suggesting the presence of subclones. We focus on these 120 samples in the following analyses. We summarize the results of these subHMM-favored samples by creating a cytoband-based stacked histogram of allele-specific SCNA events. These histograms are used to examine the frequency of allele-specific SCNA events over samples, across prespecified chromosomal locations. We found that the most frequent mainclone SCNA event is a hemizygous deletion (genotype A) on Chromosomes 3 (Figure D.1 in Appendix D of the Supplementary Materials) and a copy number gain (genotype AAB) between cytoband q21.3 and q35.3 on Chromosome 5 (Figure D.1 in Appendix D of the Supplementary Materials). Figure D.2 in Appendix D of the Supplementary Materials shows subclone SCNA events across the entire chromosomes; the most frequent subclone SCNA event is whole-genome doubling with genotype AABB (balanced copy number amplification; 15 cases out of 120 are identified as the dominant subclone genotype AABB across the entire genome) as shown in Figures D.2 and D.3 in Appendix D of the Supplementary Materials. Identifying subclone SCNA events is a unique feature of our approach and cannot be done with previously proposed methods. The high rate of balanced copy number amplification among subclonal cells may be a defining feature of the tumor type and may suggest a new target therapy. 


\section{Simulations}

We evaluate the performance of subHMM under the different clonal proportion scenario. Specifically, we investigate if the estimation of global parameters obtained at the first step is robust to an incorrect constant clonal proportion assumption that is made at this step in the two-step algorithm. We also evaluate the estimation of region-specific clonal proportions as well as subclone regions and their corresponding subclone genotypes.

All the results and summaries are based on 300 simulated datasets. Each dataset includes four subclone regions with clonal proportions $\left(M_{1}=M_{2}=0.8\right.$, $\left.M_{3}=M_{4}=0.7\right)$ and has a similar copy number profile to the result from Section 3 (the application study) based on the state space corresponding to copy number 2. First, we specifiy three different probability matrices, $P_{z^{\prime} z}^{Z}$, $P_{u^{\prime} u}^{U}$, and $P_{t}^{T}$ to form the probability transition matrix $A$. Second, we generate a sequence from a Markov chain with transition matrix $A$ of size 30,000 by using the $\mathrm{R}$ package markovchain. Given this sequences, both the expectations of $\log \mathrm{R}$ and $\log \mathrm{OR}$ are computed by using Equations 2.1 and 2.2 with the given parameters. Finally, $\log \mathrm{R}$ and $\log \mathrm{OR}$ observations are randomly generated from t- and normal distributions with means given by their expected values and measured error variances, respectively. Similar to the application, $90 \%$ of $\log \mathrm{OR}$ values are assigned as missing values. All the results and summaries are based on 300 simulated datasets.

According to Table 3, all the global estimates and corresponding Hessianbased standard errors $\left(\mathrm{SE}_{\mathrm{H}}\right)$ are close to the true values. This demonstrates that global parameter estimates and their associated Hessian-based standard errors obtained in the first step are unbiased even when the clonal proportions vary across regions. The clonal proportion estimation obtained from the second step is also unbiased (Table 4).

Furthermore, we examine the accuracy of genotypes. Figure 4 shows the probability of mainclone genotype, subclone region, and subclone genotype detection across the genome (black lines). The true clonal regions and genotypes are also shown (red lines). The figure demonstrates that subHMM does a good 
job in estimating these genomic features in realistic scenarios such as the renal cell carcinoma sample.

\section{Discussion}

In this paper, we developed a new hidden Markov model approach analyzing ASCNA that allows identifications of subclonal copy number alterations in heterogeneous tumor samples. Specifically, we use a hidden Markov model structure to locate these clonal-specific copy number alterations and their associated genotypes. Through this additional inference, our method provides further understanding of the carcinogenesis process that may ultimately lead to new cancer treatments by targeting subclonal copy number alterations of cancer driver genes.

We proposed a two-step estimation approach where we made the constant clonal proportion assumption in the first step and then estimate different proportions corresponding to identified subclone regions in the second step. Inference on the regional subclone proportions and their associated genotype is a challenging statistical problem. Specifically, when the clonal proportion varies by subclone, the emission distribution depends on the region-specific clonal proportion. In this case, the forward-backward algorithm cannot be applied, making E-step computations intractable. Inspired by this challenge, the two-step algorithm is proposed to handle this complex from intractable to tractable solution. In the first step, the standard forward-backward algorithm in HMM can evaluate the full $\log$ likelihood based on two sets of expected sufficient statistics, $\gamma_{k}$ and $\eta_{k-1, k}$, under the constant clonal proportion assumption. The proposed methodology is simple to implement and will provide practitioners the opportunity for enhanced interence in SCNA.

As illustrated in this article, our methodology can be used for a populationbased study with many samples. In our analysis, we summarized molecular profiles across samples of the same tumor. Other applications are possible, where we compare these SCNAs across tumor type, different types of exposure, or by different treatments. 


\section{Software}

Software in the form of $\mathrm{R}$ code, together with a sample input data set and complete documentation is available as a $\mathrm{R}$ package at https://dceg.cancer.gov/tools/analysis/subhmm.

\section{Supplementary Material}

Supplementary material is available in a separate file.

\section{Acknowledgments}

We would like to acknowledge our usage of the data from The Cancer Genome Atlas (TCGA) supported by the National Cancer Institute and National Human Genome Research Institute: https://cancergenome.nih.gov. We would like to thank Bill Wheeler (Information Management Services) and Lei Song (Biostatistics Branch) for computational contributions. Conflict of Interest: None declared.

\section{References}

Baum, L. E. (1972). An inequality and associated maximization thechnique in statistical estimation for probabilistic functions of markov process. Inequalities 3, 1-8.

Cheng, Y., J. Y. Dai, T. G. Paulson, X. Wang, X. Li, B. J. Reid, and C. Kooperberg (2017). Quantification of multiple tumor clones using gene array and sequencing data. The annals of applied statistics 11(2), 967.

Choo-Wosoba, H., P. S. Albert, and B. Zhu (2018). hseghmm: hidden markov model-based allele-specific copy number alteration analysis accounting for hypersegmentation. BMC bioinformatics 19(1), 424.

Egeblad, M., E. S. Nakasone, and Z. Werb (2010). Tumors as organs: complex 
tissues that interface with the entire organism. Developmental cell 18(6), 884-901.

Fan, Z., L. Mackey, et al. (2017). Empirical bayesian analysis of simultaneous changepoints in multiple data sequences. The Annals of Applied Statistics 11(4), 2200-2221.

Ha, G., A. Roth, J. Khattra, J. Ho, D. Yap, L. M. Prentice, N. Melnyk, A. McPherson, A. Bashashati, E. Laks, et al. (2014). Titan: inference of copy number architectures in clonal cell populations from tumor whole-genome sequence data. Genome research 24(11), 1881-1893.

Li, A., Z. Liu, K. Lezon-Geyda, S. Sarkar, D. Lannin, V. Schulz, I. Krop, E. Winer, L. Harris, and D. Tuck (2011). Gphmm: an integrated hidden markov model for identification of copy number alteration and loss of heterozygosity in complex tumor samples using whole genome snp arrays. Nucleic acids research 39(12), 4928-4941.

Li, Y. and X. Xie (2015). Deconvolving tumor purity and ploidy by integrating copy number alterations and loss of heterozygosity. Bioinformatics 31(4), 618.

Liu, Z., A. Li, V. Schulz, M. Chen, and D. Tuck (2010). Mixhmm: inferring copy number variation and allelic imbalance using snp arrays and tumor samples mixed with stromal cells. PloS one 5(6), e10909.

Peel, D. and G. J. McLachlan (2000). Robust mixture modelling using the t distribution. Statistics and computing 10(4), 339-348.

Shen, R. and V. E. Seshan (2016). Facets: allele-specific copy number and clonal heterogeneity analysis tool for high-throughput dna sequencing. Nucleic acids research 44(16), e131-e131.

Titsias, M. K., C. C. Holmes, and C. Yau (2016). Statistical inference in hidden markov models using k-segment constraints. Journal of the American Statistical Association 111(513), 200-215. 
Van Loo, P., S. H. Nordgard, O. C. Lingjærde, H. G. Russnes, I. H. Rye, W. Sun, V. J. Weigman, P. Marynen, A. Zetterberg, B. Naume, et al. (2010). Allelespecific copy number analysis of tumors. Proceedings of the National Academy of Sciences 107(39), 16910-16915.

Yau, C., O. Papaspiliopoulos, G. O. Roberts, and C. Holmes (2011). Bayesian non-parametric hidden markov models with applications in genomics. Journal of the Royal Statistical Society: Series B (Statistical Methodology) 73(1), 3757.

Zack, T. I., S. E. Schumacher, S. L. Carter, A. D. Cherniack, G. Saksena, B. Tabak, M. S. Lawrence, C.-Z. Zhang, J. Wala, C. H. Mermel, et al. (2013). Pan-cancer patterns of somatic copy number alteration. Nature genetics 45(10), 1134. 
bioRxiv preprint doi: https://doi.org/10.1101/675512; this version posted June 20, 2019. The copyright holder for this preprint (which was not certified by peer review) is the author/funder. This article is a US Government work. It is not subject to copyright under 17 USC 105 and is also made available for use under a CCO license.

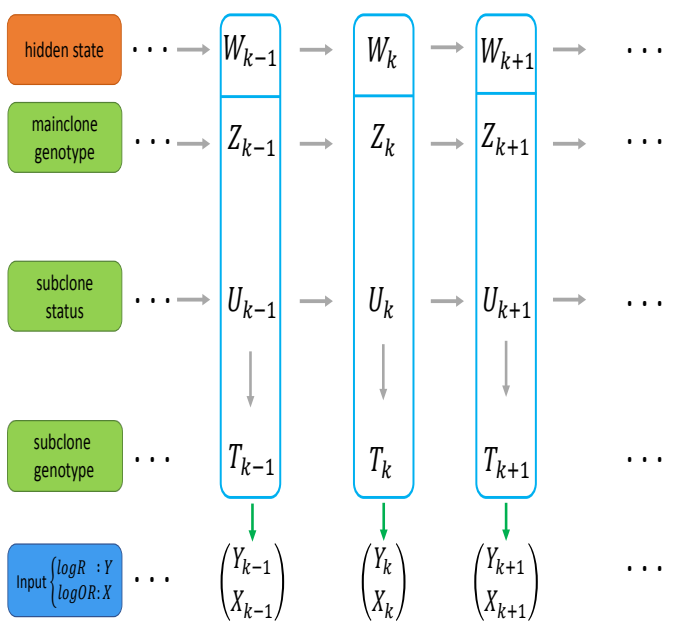

Figure 1: The transition flow chart of subHMM 

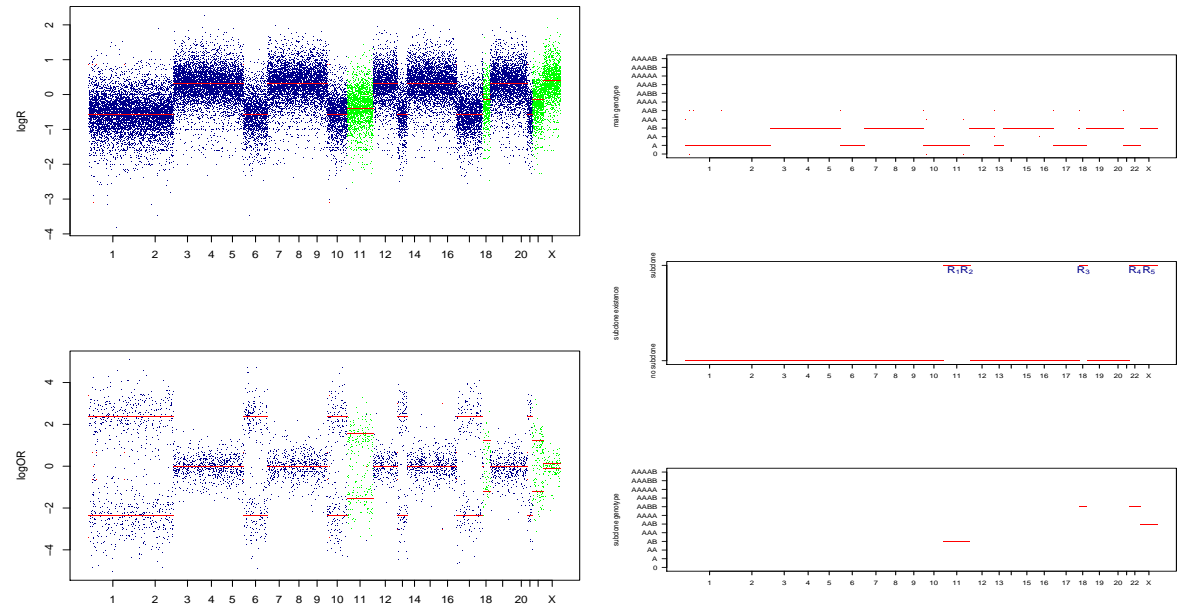

(a) The subHMM-based estimation of $\log R$

(b) The subHMM-based clonal profile and $\log \mathrm{OR}$

Figure 2: subHMM-based subclonal profile of TCGA-KL-8331; the $r$ th subclone region $R_{r}$ defines as a consecutive loci where $U_{k}=1$ with at least a length of 50 

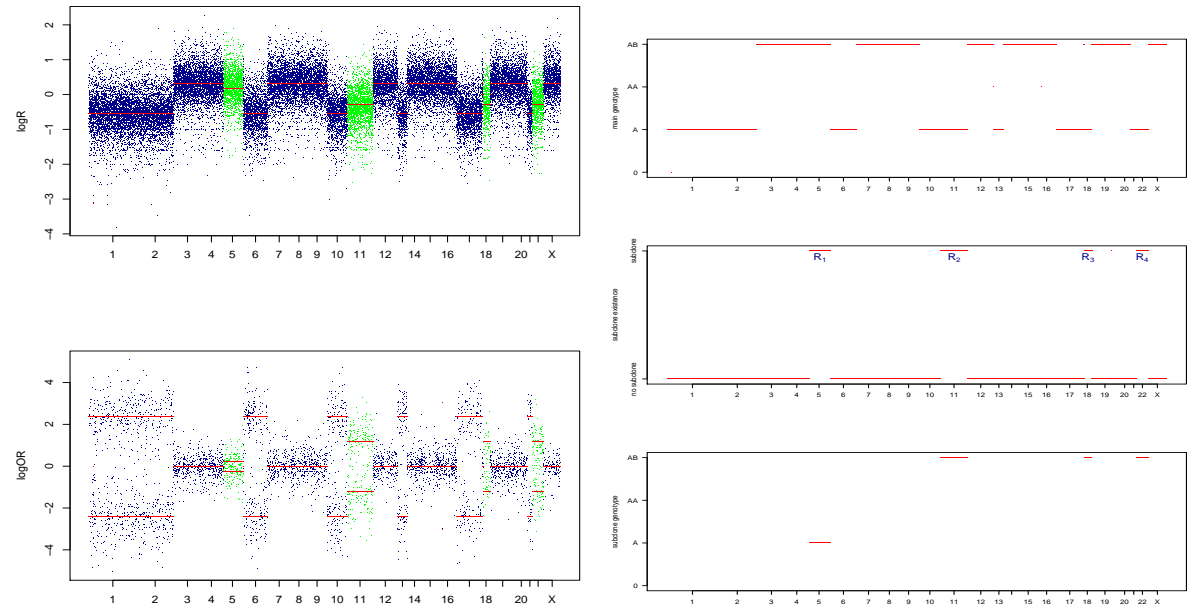

(a) The subHMM-based estimation of $\log R$

(b) The subHMM-based clonal profile and $\log \mathrm{OR}$

Figure 3: subHMM-based subclonal profile of TCGA-KL-8331 with copy number upto 2 as hidden state space; the $r$ th subclone region $R_{r}$ defines as a consecutive loci where $U_{k}=1$ with at least a length of 50 

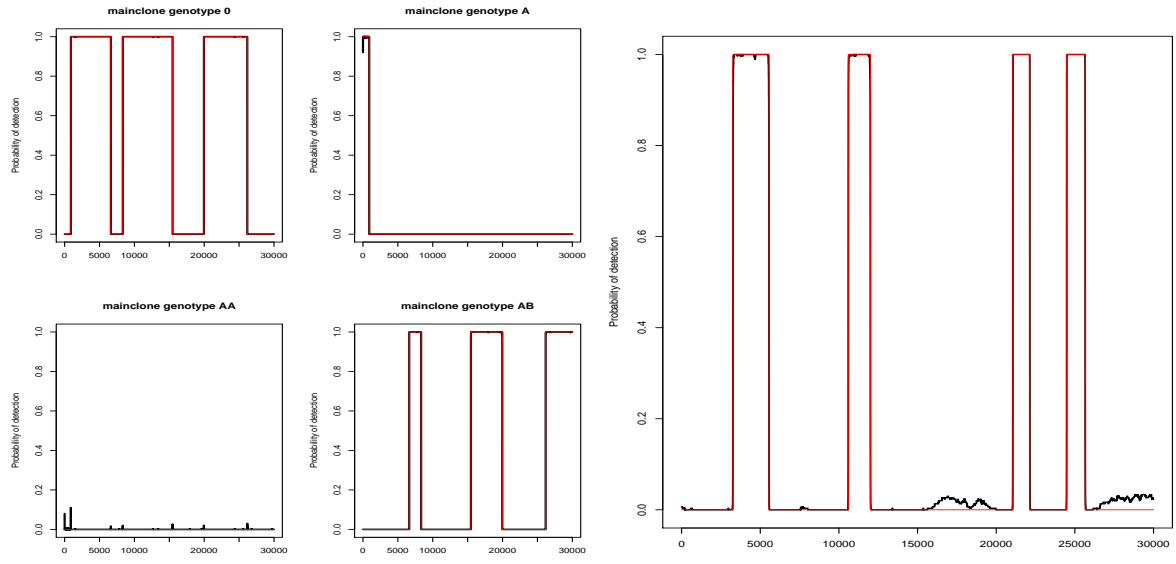

(a) mainclone genotype profile

(b) subclone region profile
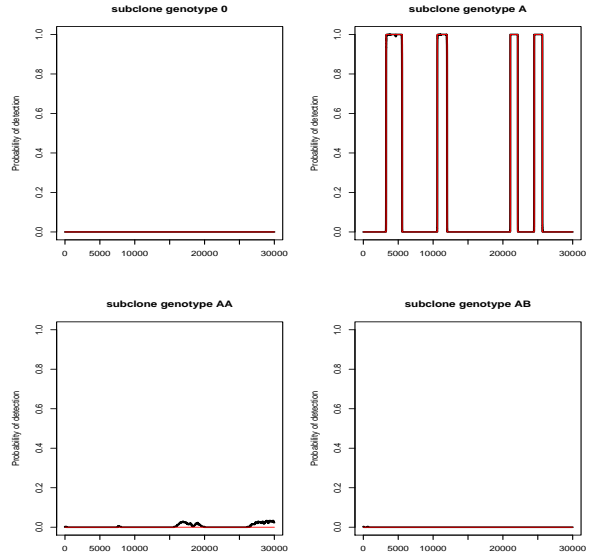

(c) subclone genotype profile

Figure 4: The probability of correct identification under the different clonal proportion assumption simulation study; The results are based on 300 simulated datasets; The red lines and black lines represent true and estimated profiles, respectively. 
Table 1: Global parameter estimation results of TCGA-KL-8331 dataset with subHMM based on state space of copy number 5

\begin{tabular}{cccccc}
\hline \multicolumn{3}{c}{ global estimation } & \multicolumn{4}{c}{ clonal proportion estimation } \\
\hline Parameters & Estimates & $\mathrm{SE}_{\mathrm{H}}$ & Parameters & Estimates & $\mathrm{SE}_{\mathrm{bs}}$ \\
$\psi$ & 1.61 & 0.0029 & $M_{1}$ & 0.89 & 0.010 \\
$\alpha$ & 0.91 & 0.0015 & $M_{2}$ & 0.88 & 0.0183 \\
$\operatorname{Var}(\log R)$ & 0.26 & 0.0033 & $M_{3}$ & 0.84 & 0.0097 \\
$\kappa^{2}$ & 0.16 & 0.0022 & $M_{4}$ & 0.84 & 0.0199 \\
$\sigma^{2}$ & 0.48 & 0.0103 & $M_{5}$ & 0.89 & 0.0111 \\
$v$ & 5.26 & 0.1550 & & & \\
\hline
\end{tabular}

Table 2: Global estimation results of TCGA-KL-8331 dataset with subHMM based on state space of copy number 2

\begin{tabular}{cccccc}
\hline \multicolumn{3}{c}{ global estimation } & \multicolumn{4}{c}{ clonal proportion estimation } \\
\hline Parameters & Estimates & $\mathrm{SE}_{\mathrm{H}}$ & Parameters & Estimates & $\mathrm{SE}_{\mathrm{bs}}$ \\
$\psi$ & 1.60 & 0.0030 & $M_{1}$ & 0.81 & 0.0085 \\
$\alpha$ & 0.91 & 0.0015 & $M_{2}$ & 0.80 & 0.0084 \\
$\operatorname{Var}(\log R)$ & 0.26 & 0.0033 & $M_{3}$ & 0.69 & 0.0173 \\
$\kappa^{2}$ & 0.16 & 0.0022 & $M_{4}$ & 0.71 & 0.0149 \\
$\sigma^{2}$ & 0.48 & 0.0105 & & & \\
$v$ & 5.27 & 0.1550 & & & \\
\hline
\end{tabular}


Table 3: Global estimation result of the different clonal proportion assumptionbased simulation study with subHMM based on 300 datasets

\begin{tabular}{ccccc}
\hline Parameters & True & Average & $\mathrm{SE}$ & $\mathrm{SE}_{\mathrm{H}}$ \\
\hline$\psi$ & 1.6 & 1.60 & 0.0069 & 0.0065 \\
$\alpha$ & 0.8 & 0.80 & 0.0012 & 0.0011 \\
$\operatorname{Var}(\log \mathrm{R})$ & 0.5 & 0.50 & 0.0063 & 0.0070 \\
$\kappa^{2}$ & 0.3 & 0.30 & 0.0040 & 0.0042 \\
$\sigma^{2}$ & 0.5 & 0.50 & 0.0148 & 0.0141 \\
$v$ & 5.0 & 5.04 & 0.1372 & 0.1452 \\
\hline
\end{tabular}

Table 4: Mainclone proportion simulation result of the different clonal proportion assumption-based simulation study with subHMM based on 300 datasets

\begin{tabular}{cccc}
\hline Parameters & True & Average & SD $^{1}$ \\
\hline$M_{1}$ & 0.8 & 0.80 & 0.0063 \\
$M_{2}$ & 0.8 & 0.80 & 0.0076 \\
$M_{3}$ & 0.7 & 0.70 & 0.0102 \\
$M_{4}$ & 0.7 & 0.70 & 0.0097 \\
\hline
\end{tabular}

${ }^{1}$ Monte-Carlo-based standard deviations 